\title{
An die Präsidentinnen und Präsidenten der in der Ärztekammer vertretenen Organisationen
}

\author{
Sehr geehrte Frau Präsidentin \\ Sehr geehrter Herr Präsident \\ Sehr geehrte Damen und Herren
}

Mit Genugtuung haben wir von den Mehrheitsentscheidungen der SGK-NR in Sachen Kontrahierungszwang Kenntnis genommen; sie entsprechen in der Mehrheit den Positionen der FMH.

Vorab ist an dieser Stelle allen zu danken, die sich durch persönliche, klinische Kleinarbeit für die Ärzteschaft verdient gemacht haben. Zu erwähnen ist insbesondere Yves Guisan, der aus relativ schwieriger Ausgangssituation entscheidend zu diesem Erfolg beigetragen hat. Das Ergebnis hat auch gezeigt, dass beharrliches, glaubwürdiges Argumentieren im persönlichen Gespräch mehr bringt als hohle millionenschwere Werbekampagnen auf Glanzpapier.

Die Entscheide lassen sich stichwortartig verkürzt wie folgt zusammenfassen:

a) Es wird nicht von einer Aufhebung des Kontrahierungszwanges, sondern von Kontrahierungszwang als Basis der Zusammenarbeit Versicherer - Arzt gesprochen.

b) Die gesetzlichen Dispositionen werden so modifiziert, dass

1. für alle Leistungen ein Qualitätsnachweis gefordert werden kann;

2. die sogenannten WZW-Kriterien - Wirksamkeit, Zweckmässigkeit und Wirtschaftlichkeit - mit Inhalt bzw. operationalisiert werden können;

3. Leistungserbringer, die den gesetzten Kriterien nicht genügen, aus den Verträgen ausgeschlossen werden können.

Es war selbstverständlich nicht möglich, die Beschlüsse - soweit überhaupt bekannt - einer Würdigung zu unterziehen; dies wird in Publikationen in der Schweizerischen Ärztezeitung geschehen.

Die Versuchung ist nun gross, sich bequem zurückzulehnen. Alle Kolleginnen und Kollegen, die solche Lüste verspüren, sei ins Stammbuch geschrieben, dass der Glaube, die ehemals goldenen Zeiten gleichsam wieder herbeisitzen zu können, der direkteste Weg in die Hölle der Fremdbestimmung durch Staat und Versicherer wäre:

- Der Nationalrat (Zweitrat!) hat noch nicht entschieden; völlig offen bleibt, was aus dem Bereinigungsverfahren mit dem Ständerat resultiert.

- Der Ärzteschaft, der FMH werden grosse Anstrengungen in Konzeptarbeit und der Wille zu realer Umsetzung abverlangt. Selbst der verwegenste Optimist würde sich hier nicht herbeilassen, auf Begeisterungsstürme in KFWB und Ärztekammer eine Bank zu verwetten.

- Die Ärzteschaft muss zu einem Konsens mit den Versicherern kommen, die in sich gespalten sind und Einigkeit darin gefunden haben, lauthals die Aufhebung des Kontrahierungszwanges zu fordern. Ob bei so diametral entgegengesetzten Positionen schon in sich a priori ein Konsens möglich ist, darf immerhin mit einigen Fragezeichen versehen werden.

- Die Politik und mit ihr der Prämienzahler wollen nun Taten sehen und nicht beschönigende Sprüche hören. Diese von den Medien geschürten Erwartungen lasten schwer auf allen Arbeiten und Verhandlungen, die wir nun unverzüglich beginnen müssen.

Mit freundlichen Grüssen

Hans Heinrich Brunner 
Sehr geehrte Frau Präsidentin

Sehr geehrter Herr Präsident

Sehr geehrte Damen und Herren

\section{Delegierte Psychotherapie}

a) Ausgangslage

TARMED 1.1, die der Urabstimmung unterbreitete und vom Bundesrat genehmigte Version, weist in Kapitel 02.03 die delegierte Psychotherapie ausschliesslich Fachärzten für Psychiatrie sowie Kinderund Jugendpsychiatern zu.

Im Rahmen der Verhandlungen haben wir zu erreichen versucht, dass auch andere Facharzttitelträger delegierte Psychotherapie anordnen können. Der erzielte Kompromiss hätte verlangt, dass Facharzttitelträger, die nicht den Titel Psychiatrie und/oder Kinder- und Jugendpsychiatrie tragen, einen Fähigkeitsausweis (FA) delegierte Psychotherapie hätten erwerben können; dabei sind wir davon ausgegangen, dass es sich um einen FA reduzierten Anforderungsprofils - eine Materie, die ohnehin einer Regelung bedarf - gehandelt hätte.

Die KWFB hat die Schaffung eines solchen FAs mit grosser Mehrheit verworfen.

\section{b) Konsequenzen/Fazit}

Die Versicherer werden nur noch delegierte Psychotherapie bezahlen, die von Facharzttitelträgern Psychiatrie und/oder Kinder- und Jugendpsychiatrie veranlasst wird.

Für die TARMED-Verhandlungsdelegation und den ZV hat sich die Sache erledigt; sie stellen den Beschluss der KWFB nicht in Frage und streichen die Problematik aus dem Verhandlungspaket TARMED-Einführungsversion.

ZV und TARMED-Verhandlungsdelegation werden nur auf einen entsprechenden anderslautenden Beschluss der Ärztekammer wieder aktiv.

\section{Dignitätserhebung}

Die Dignitätserhebung TARMED wird im ersten Quartal 2003 stattfinden; Sie werden noch detailliert über den konkreten Ablauf orientiert werden. Aus grundsätzlichen Überlegungen halten wir an dieser Stelle fest, dass die Erhebung aus rechtlichen wie operativen Gründen basierend auf Version TARMED 1.1 durchgeführt wird. Mit anderen Worten, die für die Einführungsversion erzielten Verbesserungen können nicht im Erfassungssystem berücksichtigt werden.

Die Einführungsversion wird aber auf der Website der FMH verfügbar sein und kann von den Mitgliedern dort jederzeit eingesehen und für das individuelle Ausfüllen der Erhebung mitberücksichtigt werden.

Mit freundlichen Grüssen

Hans Heinrich Brunner 